Published in final edited form as:

Biometrics. 2018 March ; 74(1): 260-269. doi:10.1111/biom.12719.

\title{
Conditional Adaptive Bayesian Spectral Analysis of Nonstationary Biomedical Time Series
}

\author{
Scott A. Bruce ${ }^{1}$, Martica H. Hall ${ }^{2}$, Daniel J. Buysse ${ }^{2}$, and Robert T. Krafty ${ }^{3,}$ \\ ${ }^{1}$ Department of Statistical Science, Temple University, Philadelphia, Pennsylvania, 19122, U.S.A \\ ${ }^{2}$ Department of Psychiatry, University of Pittsburgh, Pittsburgh, Pennsylvania, 15213, U.S.A \\ ${ }^{3}$ Department of Biostatistics, University of Pittsburgh, Pittsburgh, Pennsylvania, 15261, U.S.A
}

\section{Summary}

\begin{abstract}
Many studies of biomedical time series signals aim to measure the association between frequencydomain properties of time series and clinical and behavioral covariates. However, the time-varying dynamics of these associations are largely ignored due to a lack of methods that can assess the changing nature of the relationship through time. This article introduces a method for the simultaneous and automatic analysis of the association between the time-varying power spectrum and covariates, which we refer to as conditional adaptive Bayesian spectrum analysis (CABS). The procedure adaptively partitions the grid of time and covariate values into an unknown number of approximately stationary blocks and nonparametrically estimates local spectra within blocks through penalized splines. CABS is formulated in a fully Bayesian framework, in which the number and locations of partition points are random, and fit using reversible jump Markov chain Monte Carlo techniques. Estimation and inference averaged over the distribution of partitions allows for the accurate analysis of spectra with both smooth and abrupt changes. The proposed methodology is used to analyze the association between the time-varying spectrum of heart rate variability and self-reported sleep quality in a study of older adults serving as the primary caregiver for their ill spouse.
\end{abstract}

\section{Keywords}

Heart rate variability; Locally stationary; Replicated time series; Reversible jump Markov chain Monte Carlo; Sleep quality; Spectrum analysis; Whittle likelihood

\section{Introduction}

The frequency-domain properties of many biological time series signals, such as heart rate variability (HRV) and electroencephalography (EEG), have been found to contain valuable

\footnotetext{
*rkrafty@pitt.edu.

8. Supplementary Materials

A zip file containing Matlab code for implementing CABS and a pdf file containing Web Appendices A-D are available with this paper at the Biometrics website on Wiley Online Library. Web Appendix A is referenced in Section 2 and displays all data from the AgeWise Caregiver Study, Web Appendix B is referenced in Section 4.4 and contains details on the sample scheme, Web Appendix C is referenced in Section 5 and describes the provided Matlab code, and Web Appendix D is referenced in Section 6 presents convergence diagnostics for the analysis of the AgeWise data.
} 
information. As a result, many biomedical studies collect and analyze time series signals from multiple subjects to better understand how power spectra relate to clinical and behavioral variables. The dynamic nature of most biological processes means that these time series are rarely stationary. A method that can quantify the association between time-varying spectra and study covariates is needed to properly reflect the temporally-evolving nature of the relationship and to better understand dynamic biological processes.

The motivating study for this article seeks to quantify the association between psychological stress and self-reported sleep quality in older adults who are the primary caregiver for their spouse. In this study, HRV is observed during a night of sleep. HRV is a measure of the elapsed time between consecutive heart beats and its power spectrum provides objective measures of stress and arousal (Hall et al., 2004). Participants also completed a self-reported questionnaire, which was used to compute the Pittsburgh Sleep Quality Index (PSQI) (Buysse et al., 1989), a clinically validated summary measure of sleep quality. In quantifying the association between the time-varying spectrum of HRV and PSQI, we aim to better understand the dynamic relationship between stress levels and sleep quality.

In the clinical and biological literature, such data are commonly analyzed using an ad hoc, four stage procedure. In the first stage, time series are partitioned into prespecified, equallyspaced intervals (e.g. two minute epochs). In the second stage, collapsed measures within prespecified frequency bands that quantify specific characteristics of heart rate variability are computed for each interval (Burr, 2007). For example, normalized high frequency (HFnu), which is inversly related to experimental stress (Hall et al., 2004), is calculated as the total power from high frequencies $(0.15-0.4 \mathrm{~Hz})$ divided by total power from high and low frequencies $(0.04-0.4 \mathrm{~Hz})$. In the third stage, interval specific measures are averaged across time to obtain time-invariant measures for each subject. Finally, in the last stage, the association between these temporally averaged measures and clinical and behavioral variables are assessed using standard tools such as Pearson's correlations and ANOVA (Malik et al., 1996; Hall et al., 2004). There are several serious drawbacks to this ad hoc approach. First, time series are segmented a priori into intervals without regard for the dynamics of the series. Second, averaging spectral measures of a given subject over time intervals inhibits the ability to assess dynamic relationships between spectral measures and other study variables. Lastly, the final stage treats the estimated spectral measures as known parameters, thus ignoring the variability accumulated through the multiple stages of the estimation procedure and leading to inaccurate inference.

In the statistics literature, many models and methods for the spectral analysis of nonstationary time series have been studied. These include methods with piecewise stationary estimators (Adak, 1998; Ombao et al., 2005; Davis et al., 2006), methods that smooth across time to obtain slowly varying estimators (Dahlhaus, 1997; Guo et al., 2003; Qin and Wang, 2009), and methods that provide estimators that can adapt to both abrupt and slowly varying temporal dynamics (Rosen et al., 2012; Yang et al., 2016). These methods follow traditional time series analysis that focus on analyzing a single time series, while methods for the analysis of a collection of time series from multiple subjects whose spectra are associated with study covariates are few and preliminary. In the stationary setting, parametric (Diggle and Al Wasel, 1997) and semi-parametric approaches (Krafty et al., 
2011) for the analysis of replicated time series have been explored. In the nonstationary setting, Qin et al. (2009) introduces a semi-parametric approach that nonparametrically smooths across time and assumes a parametric linear relationship between log-spectra and covariates. Fiecas and Ombao (2016) propose a nonparametric approach to estimating the time- and covariate-varying spectrum that circumvents the parametric assumptions of Qin et al. (2009) by smoothing across covariate. However, the approaches of both Qin et al. (2009) and Fiecas and Ombao (2016) are formulated for spectra that evolve continuously and smoothly as functions of both time and covariate. In many applications, the spectral properties of a time series may also change suddenly. For instance, in our motivating study, there might be smooth trends in HRV spectra throughout the night, but abrupt changes are expected when the body transitions between different sleep stages. To the best of our knowledge, there currently are no formal statistical methods that allow for the analysis of associations between covariate and time-varying power spectra that can capture both abrupt and slowly varying changes with respect to time and covariate.

This article proposes a method for simultaneous and automatic estimation of the association between the time-varying spectrum and covariate, which we refer to as conditional adaptive Bayesian spectrum analysis, or CABS. A covariate-indexed locally-stationary model is presented, in which, similar to Fiecas and Ombao (2016), spectra are nonparametric functions of frequency, covariate, and time. Unlike Fiecas and Ombao (2016), the proposed model allows for discontinuous changes in time and covariate. The model is formulated in a Bayesian framework where the number and location of time and covariate partition points are random variables and fit using reversible-jump Markov chain Monte Carlo (RJMCMC) techniques. CABS provides a flexible and adaptive estimator of the time- and covariatevarying spectrum that broadens the existing scope of processes and scientific questions that can be addressed in three important ways. First, the approach uses the data to determine the appropriate number and location of time and covariate partition points rather than requiring a prespecified segmentation scheme for the locally-stationary model. Second, by averaging over the posterior distribution of the number and locations of time and covariate partition points, the estimator can recover both smooth and abrupt changes in the spectrum across time and covariate. Finally, the sampling procedure naturally enables inference on any function of the time and covariate-varying spectrum, including collapsed measures through time, and the location and magnitude of abrupt changes, both of which are of importance in applied analyses.

This paper is organized as follows. Section 2 provides more details about the motivating study: the AgeWise Caregiver Study. Section 3 presents the specification of the covariateindexed time-dependent power spectrum. The CABS estimation procedure for the covariateindexed time-dependent power spectrum is detailed in Section 4. Simulation results for slowly and abruptly changing spectra are presented in Section 5, and the proposed method is applied to the AgeWise Caregiver Study in Section 6. Concluding remarks are found in Section 7. 


\section{Motivating Study}

Older adults who are the primary caregiver for their ill spouse often experience significant mental and emotional stress, and are likely to develop some form of sleep disturbance that can negatively impact health and functioning (McCurry et al., 2007). A goal of the AgeWise Caregiver Study conducted at the University of Pittsburgh was to gain a better understanding of the association between stress and sleep in older adults who were the primary caregiver for their ill spouse.

The current analysis considers data from 30 men and women 60-89 years of age. Each participant served as the primary caregiver for their spouse who was suffering from a progressive dementing illness such as Alzheimer's or advanced Parkinson's disease. As previously mentioned, study participants completed a self-reported questionnaire used to formulate a PSQI score (Buysse et al., 1989). The PSQI score is a popular clinical measure of self-reported sleep disturbances and of how these disturbances affect daily functioning over a one-month period. PSQI scores can range in value from 0-21; larger scores represent more disturbed sleep, with scores of 6 or larger typically taken as an indicator of clinically disturbed sleep. In our sample, PSQI scores ranged from 1-13 and had a mean of 7.

Participants were also studied during a night of in-home sleep through ambulatory polysomnography (PSG), which is the comprehensive recording of electrophysiological changes during sleep. The PSG used in the study included an electrocardiograph (ECG) to monitor heart activity. The ECG was used to locate the timing of heart beats, which were then differenced, detrended, linearly interpolated, and resampled at $1 \mathrm{~Hz}$ to compute a HRV series. During the night, the body cycles through two types of sleep: non-rapid eye movement (NREM) sleep, which contains deep sleep, and rapid eye movement (REM) sleep, in which dreaming typically occurs. We isolated a 10 minute long epoch of HRV for each participant during the 5 minutes before and 5 minutes after the first onset of REM sleep.

In healthy individuals, the spectrum of $\mathrm{HRV}$ is expected to change as subjects move from NREM to REM. Disruptions in these patterns have been observed under stress and represent a mechanism through which stress may negatively affect health and functioning (Hall et al., 2004). We desire an analysis of the association between the time-varying spectrum of HRV and PSQI to quantify how the dynamics of HRV spectra during the transition from NREM to REM are associated with sleep quality. HRV time series and PSQI scores for two subjects are displayed in Figure 1 to illustrate study data. HRV time series and PSQI scores for all participants can be found in Web Appendix A.

\section{Covariate-Indexed Time-Dependent Spectrum}

We consider modeling a collection of time series $X_{\ell t}$ of length $t=1, \ldots, T$ and covariates w $\in[0,1]$ from $\ell=1, \ldots, L$ independent subjects as covariate-indexed locally stationary processes. Formally, we consider the model 


$$
X_{\ell t}=\int_{-1 / 2}^{1 / 2} A\left(t / T, w_{\ell}, \nu\right) \exp (2 \pi i t \nu) d Z_{\ell}(\nu)
$$

where $Z_{\ell}$ are independent and identically distributed orthogonal processes and $A(u, w, v)$ is a complex-valued function of scaled time $u \in[0,1]$, covariate $w \in[0,1]$, and frequency $v \in$ $\mathbb{R}$ such that $A(u, w, v)=A(u, w, v+2 \pi)$ and $A(u, w, \nu)=\overline{A(u, w,-\nu)}$. This extension of the time-varying Cramér representation (Priestley, 1965) allows the transfer function to vary not only across frequency and time, but also across covariate.

The covariate-indexed time-dependent power spectrum is then defined as $f(u, w, v)=\mid A(u$, $w, v)\left.\right|^{2}$. The power spectrum $f(u, w, v)$ can be interpreted as the contribution to the conditional variance at time $u T$, conditional on covariate $w$, from oscillations at frequency $v$. We assume that $A$, and subsequently the spectrum $f$, are continuous functions of frequency $v$, but can have a finite number of discontinuities as functions of scaled time $u$ and covariate $w$. This flexibility allows for modeling abrupt changes in time and covariate.

It should be noted that this definition can be viewed as an extension of the piecewise locally stationary model proposed by Adak (1998), where $f(u, v)$ is continuous in $v$ but can be discontinuous in $u$. This differs from the locally stationary models considered by Dahlhaus (1997) and Guo et al. (2003), which require continuity in both $u$ and $v$ so that smoothing can be used to obtain consistent estimators.

\section{CABS: Conditional Adaptive Bayesian Spectrum Analysis}

\subsection{Piecewise Stationary Approximation}

Our analysis procedure, CABS, begins by noting that the covariate-indexed locally stationary model can be approximated by piecewise stationary processes via partitioning the plane of time-covariate values into approximately stationary blocks. A partition of the time and covariate space into $m$ time-based segments and $p$ covariate-based segments is denoted by $\boldsymbol{\xi}_{m}=\left(\xi_{0}, \ldots, \xi_{m}\right)^{\prime}$ and $\psi_{p}=\left(\psi_{0}, \ldots, \psi_{p}\right)^{\prime}$, where $\xi_{j}$ is the unknown location of the end of the $j$ th time segment and $\psi_{g}$ is the unknown location of the end of the gth covariate segment. Conditional on $m, p, \boldsymbol{\xi}_{\boldsymbol{m}}$, and $\psi_{\boldsymbol{p}}$,

$$
X_{\ell t} \approx \sum_{j=1}^{m} X_{\ell t}^{(j, g)} \delta_{j}(t) \delta_{g}\left(w_{\ell}\right)
$$

where for $j=1, \ldots, m$ and $g=1, \ldots, p$, the processes $X_{\ell t}^{(j, g)}$ are stationary with power spectrum $f_{j, g}(v)$, and $\delta_{j}(t), \delta_{g}(w \theta)$ are indicator functions such that $\delta_{j}(t)=1$ if $t \in\left(\xi_{j-1}, \xi_{j}\right]$ and 0 otherwise, and $\delta_{g}(w \ell)=1$ if $w \ell \in\left(\psi_{g-1}, \psi_{g}\right]$ and 0 otherwise. See Figure 2 for an illustration of a partition of the time and covariate space. 


\subsection{Local Spectrum Estimation}

Given a partition of the time and covariate space $\left(\xi_{m}, \psi_{p}\right)$, let

$$
Y_{j, \ell}(\nu)=\frac{1}{T_{j}}\left|\sum_{t=1}^{T} \delta_{j}(t) x_{\ell t} \exp (-2 \pi i \nu t)\right|^{2}
$$

be the local periodogram for the Ah subject within time block $j$, which contains $T_{j}=T\left(\xi_{j}-\right.$ $\left.\xi_{j-1}\right)$ time points. The periodogram is an unbiased but noisy estimator of the true spectrum and can be smoothed across frequency to construct a consistent estimator. Efficient estimators can be obtained by utilizing the large sample distribution of the periodogram (Pawitan and O'Sullivan, 1994). For each time segment $j$, let $n_{j}=\left\lfloor T_{j} / 2\right\rfloor-1$ and $v_{j k}=k / T_{j}$ for $k=1, \ldots, n_{j}$ be the Fourier frequencies. Under appropriate conditions, for large $T_{j}(j=1$, ..., $m$ ), the overall likelihood can be approximated by a product of local Whittle likelihoods (Whittle, 1957)

$$
\begin{gathered}
L\left(f_{1,1}, \ldots, f_{m, p} \mid \mathbf{x}, \boldsymbol{\xi}_{m}, \boldsymbol{\psi}_{p}\right) \approx \prod_{\ell=1}^{L} \prod_{g=1}^{p} \prod_{j=1}^{m}(2 \pi)^{-n_{j} / 2} \\
\prod_{k=1}^{n_{j}} \times \exp \left\{-\delta_{g}\left(w_{\ell}\right)\left[\log f_{j, g}\left(\nu_{j k}\right)+Y_{j, \ell}\left(\nu_{j k}\right) / f_{j, g}\left(\nu_{j k}\right)\right]\right\} .
\end{gathered}
$$

We consider a Bayesian penalized spline model on the local log spectrum within each stationary block for unconstrained estimation. Linear splines are used as they have been found to better capture changes in the power spectra compared to higher order splines (Rosen et al., 2012). The local log spectrum is modeled as

$$
\log f_{j, g}(\nu) \approx \alpha_{j, g}+\sum_{b=1}^{B} \beta_{b}^{(j, g)} \cos (2 \pi b \nu)
$$

The functions $\cos (2 \pi b v)$ are the Demmler-Reinsch basis for linear, periodic, even splines when data are observed on an evenly spaced grid (i.e. the Fourier frequencies) (Schwarz and Krivobokova, 2016). We define the $n_{j} \times B$ matrix of the basis functions evaluated at the Fourier frequencies as $\mathbf{Z}_{j}$ where $\left\{Z_{j}\right\}_{k, b}=\cos \left(2 \pi b v_{j k}\right)$. Prior distributions are assumed on the coefficients such that $\beta^{(j, g)}=\left(\beta_{1}^{(j, g)}, \ldots, \beta_{B}^{(j, g)}\right)^{\prime} \sim N\left(0, \tau_{j, g}^{2} D_{B}\right)$, where $D_{B}=$ $\operatorname{diag}\left(\{2 \pi b\}^{-2}\right)$, which is independent of $\alpha_{j, g} \sim N\left(0, \sigma_{\alpha}^{2}\right)$. The smoothing parameter, $\tau_{j, g}^{2}$, controls the roughness of the $\log$ spectrum, and a uniform prior is placed on $\tau_{j, g}^{2}$ such that $p\left(\tau_{j, g}^{2}\right)=1 / \tau_{j, g}^{2}$. The hyperparameter $\sigma_{\alpha}^{2}$ is set to be a large, fixed number. 
A two-step sampling scheme for the parameters $\boldsymbol{a}_{j, g}, \boldsymbol{\beta}^{(j, g)}$, and $\tau_{j, g}^{2}$ using MCMC methods can be constructed for estimation as follows (Rosen et al., 2012).

1. Given a set of realizations of the local $\log$ periodogram, $\log \left(\mathbf{Y}_{j, g}\right)=\left\{\log \left[Y_{j, \ell}\right.\right.$ $\left.\left(v_{j k}\right)\right]: w_{\ell} \in\left(\psi_{g-1}, \psi_{g}\right]$ and $v_{j k}=k / T_{j}$ for $\left.k=1, \ldots, n_{j}\right\}$ and basis functions $\mathbf{Z}_{j}$, $a_{j, g}$ and $\beta^{(j, g)}$ are sampled jointly in a Metropolis-Hastings (M-H) step from

$$
\begin{gathered}
p\left(\alpha_{j, g}, \boldsymbol{\beta}^{(j, g)} \mid \tau_{j, g}^{2}, \log \left(\boldsymbol{Y}_{j, g}\right), \boldsymbol{Z}_{j}\right) \propto \\
\exp \left\{-\sum_{\ell=1}^{L} \sum_{k=1}^{n_{j}} \delta_{g}\left(w_{\ell}\right)\left[\alpha_{j, g}+\mathbf{z}_{j k}^{\prime} \boldsymbol{\beta}^{(j, g)}+\exp \left(\log \left[Y_{j, \ell}\left(\nu_{j k}\right)\right]-\alpha_{j, g}-\mathbf{z}_{j k}^{\prime} \boldsymbol{\beta}^{(j, g)}\right)\right]-\frac{\alpha_{j, g}}{2 \sigma_{a}^{2}}-\frac{1}{2 \tau_{j, g}^{2}} \boldsymbol{\beta}^{(j, g)^{\prime}} D_{B}^{-1} \boldsymbol{\beta}^{(j, g)}\right\},
\end{gathered}
$$

where $\mathbf{z}_{j k}^{\prime}$ is the $k$ th row of $\mathbf{Z}_{j}$.

2. $\tau_{j, g}^{2}$ is sampled from the inverse gamma distribution with density

$$
p\left(\tau_{j, g}^{2} \mid \boldsymbol{\beta}^{(j, g)}\right) \propto\left(\tau_{j, g}^{2}\right)^{-B / 2} \exp \left(-\frac{1}{2 \tau_{j, g}^{2}} \boldsymbol{\beta}^{(j, g)^{\prime}} D_{B}^{-1} \boldsymbol{\beta}^{(j, g)}\right)
$$

\subsection{Adaptive Partition Estimation}

To complete the Bayesian model specification, we specify priors on the number and location of time and covariate partition points. This allows the number and location of partition points to be adaptively estimated from the data, so both smooth and abrupt changes to the spectrum can be captured by averaging over the posterior distribution of the partition.

Following Rosen et al. (2012), discrete uniform priors are used for the number of time and covariate segments such that $\operatorname{Pr}(m=k)=1 / M$ for $k=1, \ldots, M$ and $\operatorname{Pr}(p=g)=1 / P$ for $g=1$, ..., $P$ for some maximum values $M$ and $P$. Given a minimum number of time points per segment $t_{\min }$, which is chosen to assure that the local Whittle likelihood provides a close approximation to the true likelihood within each block, conditional on the number of segments, constrained uniform priors are placed on segment end points

$$
\begin{gathered}
\operatorname{Pr}\left(\boldsymbol{\xi}_{m} \mid m\right)=\prod_{j=1}^{m-1} \operatorname{Pr}\left(\xi_{j} \mid \xi_{j-1}, m\right) \\
\operatorname{Pr}\left(\boldsymbol{\psi}_{p} \mid p\right)=\prod_{g=1}^{p-1} \operatorname{Pr}\left(\psi_{g} \mid \psi_{g-1}, p\right)
\end{gathered}
$$


where $\operatorname{Pr}\left(\xi_{j}=t \mid m\right)=1 / p_{j, m}, \operatorname{Pr}\left(\psi_{g}=w \mid p\right)=1 / p_{g, p}, p_{j, m}$ is the number of possible locations for the endpoint of the $j$ h segment subject to the constraint that at least $t_{\min }$ observations are contained in each of the $m$ segments, and $p_{g, p}$ is the number of possible locations for the endpoint of the gth segment such that at least one covariate value is contained in each of the $p$ segments.

\subsection{Sampling Scheme}

A reversible jump Markov chain Monte Carlo sampler is presented here for flexible and adaptive estimation of the time and covariate-varying spectrum. Each MCMC iteration conducts two types of moves, within-model moves and between-model moves, that may alter the time and covariate partitions separately and in turn. A description of the sampling scheme is given here while technical details are provided in Web Appendix B. Superscripts $c$ and $p$ denote current and proposed parameter values, respectively.

4.4.1 Within-Model Moves-Given the current partition of the time and covariate space, $\left(\xi_{m} c, \psi_{p} c\right)$, a single time partition point, $\xi_{j *}$, is proposed to be relocated. Across all covariate segments, the corresponding basis function coefficients in the pair of adjacent time segments impacted by the relocation, $\left[\boldsymbol{\beta}^{\left(j^{*}, g\right)}, \boldsymbol{\beta}^{\left(j^{*}+1, g\right)}\right]$ for $g=1, \ldots, p^{c}$, are updated. These two steps are jointly accepted or rejected in a M-H step. The smoothing parameters are then updated in a Gibbs step according to Equation (7). Then, the same process is carried out to consider relocation of one of the covariate partition points, $\psi_{g}$ * The impacted basis functions, $\left[\boldsymbol{\beta}^{\left(j, g^{*}\right)}, \boldsymbol{\beta}^{\left(j, g^{*}+1\right)}\right]$ for $j=1, \ldots, m^{c}$, are updated and accepted or rejected in a similar M-H step followed by a similar update to the smoothing parameters.

4.4.2 Between-Model Moves-The number of time segments is either proposed to increase by $1\left(m^{p}=m^{c}+1\right)$ or decrease by $1\left(m^{p}=m^{c}-1\right)$.

- If a birth is proposed $\left(m^{p}=m^{c}+1\right)$, a time segment is selected for splitting and an additional partition point is selected from within this segment. For each of the covariate segments, two new smoothing parameters are created from the current single parameter. Then, new sets of basis function coefficients are drawn conditional on the new smoothing parameters and the move is accepted or rejected in a M-H step.

- If a death is proposed $\left(m^{p}=m^{c}-1\right)$, a time partition point is selected for removal. For each of the covariate segments, a single smoothing parameter is formed from the two currently adjacent smoothing parameters. Similarly, new sets of basis function coefficients are drawn conditional on the new smoothing parameters and the move is accepted or rejected in a M-H step.

This process is then repeated similarly for the covariate partition resulting in a potential birth $\left(p^{p}=p^{c}+1\right)$ or death $\left(p^{p}=p^{c}-1\right)$ in the number of covariate segments.

\section{Simulations}

To better illustrate the proposed methodology and the flexibility of our approach to capture both smooth and abrupt changes, we present results from a simulated piecewise AR process 
and a simulated slowly varying AR process, both modulated by a covariate. CABS is fit to each simulated data using 5,000 total iterations with the first 1,000 used as a burn-in. The maximum number of time and covariate segments is set to $M=10$ and $P=8, t_{\min }$ is set to 50 , and the number of spline basis functions is set to $B=7$.

\subsection{Piecewise AR Process}

We consider a collection of $L=8$ conditional piecewise AR processes of length $T=1000$ with covariates $w \ell=(\ell-1) /(L-1)$ for $\ell=1, \ldots, L$ where

$$
x_{\ell t}= \begin{cases}-\phi_{\ell} x_{\ell t-1}+\varepsilon_{\ell t}, & \text { for } 1 \leq t \leq 500 \\ +\phi_{\ell} x_{\ell t-1}+\varepsilon_{\ell t}, & \text { for } 501 \leq t \leq 1000\end{cases}
$$

$$
\phi_{\ell}= \begin{cases}0.5 & \text { for } 0 \leq w_{\ell} \leq 0.5 \\ 0.9 & \text { for } 0.5<w_{\ell} \leq 1\end{cases}
$$

and $\varepsilon_{\ell t} \stackrel{\text { iid }}{\sim} N(0,1)$. The spectrum has a single abrupt change in time, a single abrupt change in the covariate, and is stationary otherwise.

The posterior distribution of the number and locations of segments can be used to evaluate the presence and location of abrupt changes. The posterior probability for $m=2$ time segments is estimated as $99.9 \%$, and the posterior mean for the location of the end of the first time segment, conditional on two time segments, is $\hat{\xi}_{1}=499.5$. Figure 3 shows the posterior distribution for the number of time segments as well as the distribution of time partition points conditional on two time segments for the piecewise AR process. Note that both distributions are tightly packed around the true number of time segments $(m=2)$ and location of the true partition point $(t=500)$. The posterior probability for $p=2$ covariate segments is $99.9 \%$ with a posterior mean for the end of the first segment, conditional on two covariate segments, of $\hat{\psi_{1}}=0.4286$. The covariate value of 0.4286 represents the largest realized value less than the true partition point of 0.5 . Figure 4 displays the true and estimated average time-varying log spectrum.

Another important consideration for estimation of the time- and covariate-varying spectrum is in how accuracy changes as more equally spaced samples are available in scaled time and scaled covariate. Table 1 contains the mean and standard deviation of the distribution of mean squared error (MSE) for 100 random samples at various sample size settings in scaled time and scaled covariate. For comparison, an ad hoc procedure similar to the one described in the introduction, but not collapsed within frequency bands to facilitate comparison, is also used to estimate the spectrum with MSE reported in Table 1. This ad hoc procedure estimates the time-varying power spectrum separately for each subject by dividing the time series into $\lfloor 50 \sqrt{T / 1000}\rfloor$ equally sized bins, where $T=1000,2000,4000$ is the number of equally spaced samples in scaled time, and then estimating the local spectrum using 
smoothing splines with penalty parameter selected by generalized cross validation following Section 3.4.2 of Gu (2013). While both methods see improved accuracy with higher sampling rates in scaled time and scaled covariate, the CABS estimator is able to more accurately estimate the spectrum by allowing for efficient information sharing across time and covariate.

\subsection{Slowly Varying AR Process}

In this section, we consider a collection of $L=8$ slowly varying AR processes of length $T=$ 1000 that are modulated by covariates $w \ell=(\ell-1) /(L-1)$ for $\ell=1, \ldots, L$ where

$$
\begin{gathered}
x_{\ell t}=\phi_{\ell t} x_{\ell t-1}+\varepsilon_{\ell t}, \quad \text { (12) } \\
\phi_{\ell t}= \begin{cases}-0.5+t / 1000 & \text { for } 0 \leq w_{\ell} \leq 0.5 \\
-0.9+9 t / 5000 & \text { for } 0.5<w_{\ell} \leq 1,\end{cases}
\end{gathered}
$$

and $\varepsilon_{\ell t} \stackrel{\text { iid }}{\sim} N(0,1)$. In this example, given a covariate value, spectra change smoothly in time.

Figure 3 contains the posterior distribution for the number of time segments for the slowly varying AR process. Note that more time segments are proposed compared to the piecewise AR process as we are using a piecewise model approximation to the slowly varying process. Figure 3 also shows how the distribution of time partition points changes from one iteration to the next for iterations with six time segments, which has the highest posterior probability. Our proposed approach can recover slowly varying processes because the spectral estimator averages over the possible locations of the time partition points reflected in the posterior distribution. By contrast, the distribution of the time partition points for the piecewise AR process is tightly packed around the true partition point, so we are averaging across a much smaller range of possible partition points. Two covariate segments with a covariate partition point at the largest realized value less than 0.5 is estimated with probability $99.9 \%$, and Figure 4 displays the true and estimated average time-varying log spectrum.

All computations were performed in MATLAB 2016b on a desktop machine with 64 GB RAM, an eight core, $3.5 \mathrm{GHz}$ processor, and a Windows 10 operating system. Run times for the complete MCMC sampler for each random sample of simulated data ranged from 12 to 15 minutes. MATLAB code for implementing the CABS procedure is provided as a zip file in Supplementary Material and is described in Web Appendix C.

\section{Application to AgeWise Caregiver Study}

We now present analytical results of applying the proposed method to the motivating study described in Section 2, which consists of HRV data for the 5 minutes before and 5 minutes after entering the first period of REM sleep and associated PSQI scores from $L=30$ study participants. To ensure proper inference, we constructed convergence diagnostics based on 5 
independent chains of length 50,000 with over-dispersed starting points (Castelloe and Zimmerman, 2002). The chains appear to reach convergence after 15,000 iterations, so we have discarded the first 15,000 iterations across all chains and pooled the remaining iterations for inference. Technical details of the convergence assessment method and results can be found in Web Appendix D.

\subsection{Time-Varying Spectra}

The proposed procedure identified one clear temporal partition point 2.3 minutes before the onset of REM with a posterior probability of $99.9 \%$. In our study, sleep staging was determined by trained technicians who inspected the electroencephalogram (EEG) following established guidelines (Iber et al., 2007). Consequently, our results indicate that changes in autonomic nervous system activity, as measured by HRV, precede neurological changes, as measured by EEG. This finding is not completely unexpected, as preliminary results have been reported in which modifications in cardiac vagal activity precede changes in the EEG power spectrum (Jurysta et al., 2003).

With regards to PSQI, the procedure separated the data into 4 segments: PSQI from 1-3, 4 $6,7-10$ and $11-13$ with a posterior probability of $99.9 \%$. Recalling that PSQI greater than 6 is typically indicative of clinically disturbed sleep, we refer to these groups as excellent, good, fair and poor sleep quality, respectively. The covariate-indexed time-varying spectrum is a 3-dimensional hypersurface, which presents challenges for visualization. However, conditional average time-frequency surfaces obtained by averaging the spectrum over a range of covariate values can elucidate the information contained in the covariate-indexed time-varying spectrum. They are particularly useful when the analysis indicates a high posterior probability for a particular partition of the covariate space, as is the case with PSQI in this application, in which averaging over the identified covariate values within each segment still provides an accurate representation of the conditional time-varying spectrum for each covariate value.

Figure 5 presents the estimated average time-varying log spectra for subjects in the two extreme groups: excellent and poor sleep quality. There are two noticeable differences between these two time-varying spectra. First, power in low frequencies (LF) from 0.04$0.15 \mathrm{~Hz}$ increases between NREM and REM for participants with excellent sleep quality, but decreases for poor sleep quality. Second, the change in power from NREM to REM for high frequencies (HF) between $0.15-0.40 \mathrm{~Hz}$ is less drastic for participants with poor sleep quality than it is for those with excellent sleep quality.

Relative power within these HF and LF bands is used by researchers as measures of autonomic nervous system activity, which is believed to play an important role in the rejuvenating properties of sleep (Vanoli et al., 1995). Consequently, differences in the dynamics of HF and LF power between people with excellent and poor sleep quality may explain how poor sleep is associated with ill-health. A favorable property of the proposed method is that the RJMCMC sampler provides a means to estimate and conduct inference on any function of the covariate-indexed time-varying spectrum. In the following subsection, we use this ability to conduct inference on the association between PSQI and these collapsed measures. 


\subsection{Collapsed Measures}

The parasympathetic branch of the autonomic nervous system is responsible for bodily activities that occur while at rest and its modulation is inversely related to stress and arousal. The amount of HF power relative to the amount of combined power from HF and LF bands, which is referred to as normalized-HF (HFnu), provides a measure of parasympathetic modulation (Malik et al., 1996). From the covariate-indexed time-varying spectrum, we can define HFnu as a function of PSQI and time

$$
\operatorname{HFnu}(u, w)=\left[\int_{0.15}^{0.40} f(u, w, \nu) d \nu\right] /\left[\int_{0.04}^{0.40} f(u, w, \nu) d \nu\right] .
$$

As previously mentioned, the method automatically provides point estimates and credible intervals for any function of the conditional power spectrum, including HFnu, through the mean and percentiles of the sample generated from the MCMC algorithm. Average HFnu( $u$, W) within each of the time segments for each of the covariate segments provides a closer exploration of this dynamic relationship. For those with excellent sleep quality (PSQI 1-3), the estimated HFnu and 95\% pointwise credible intervals in NREM and REM are 0.27 $(0.21,0.34)$ and $0.21(0.17,0.24)$, respectively. For those with poor sleep quality (PSQI 1113), the estimated HFnu and 95\% pointwise credible intervals in NREM and REM are 0.09 $(0.07,0.11)$ and $0.15(0.13,0.17)$, respectively.

These point estimates and credible intervals show that HFnu is significantly higher across the entire period of observation for those with excellent sleep quality as compared to those with poor. Further, they show that there is a significant decrease in HFnu from NREM to REM in participants with excellent sleep, which is expected in healthy individuals (Vanoli et al., 1995), but that there is a significant increase in those with poor sleep quality. Our results suggest that not only do individuals with poor sleep quality exhibit increased autonomic nervous system arousal during sleep, but the dynamics of their parasympathetic activity across sleep periods differs from good sleepers, representing two potential pathways through which poor sleep quality may be linked to ill-health and functioning.

\section{Concluding Remarks}

This article proposes a new method, CABS, for the simultaneous and automatic analysis of the association between the time-varying spectrum and study covariates. CABS is one of the first approaches to analyzing the power spectrum of replicated nonstationary time series indexed by a covariate and is not meant to be plenary. We conclude this article by discussing three limitations and related future extensions. First, the covariate-indexed locally stationary model assumes that time series with the same covariate have the same time-varying spectrum, while extra spectral variability may be present in some applications (Krafty, 2016). A topic of future research would be to incorporate within-group spectral variability into the modeling framework. Second, the presented approach considers the impact of a single static covariate on the time-varying spectrum and can be extended to include multiple fixed or time-varying covariates. Incorporating multiple and time-varying covariates would require some form of simultaneous dimension reduction to avoid exponential increases in 
computational complexity. Lastly, the local Whittle likelihood requires there to be enough time points within approximately stationary blocks $\left(t_{\min }\right)$ for the central limit theorem to ensure a good approximation to the large sample distribution of local periodograms. If two abrupt changes occur in close proximity relative to the sampling rate, this might not be possible. Future work will examine leveraging information across replicates to circumvent this potential limitation.

\section{Supplementary Material}

Refer to Web version on PubMed Central for supplementary material.

\section{Acknowledgments}

This work is supported by NIH grants R01GM113243, P01AG20677 and R01HL104607, and NSF grant CNS0958854. The authors thank the Editor, Associate Editor and two Referees for insights and comments that greatly improved the manuscript.

\section{References}

Adak S. Time-dependent spectral analysis of nonstationary time series. Journal of the American Statistical Association. 1998; 93:1488-1501.

Burr RL. Interpretation of normalized spectral heart rate variability indices in sleep research: A critical review. Sleep. 2007; 30:913-919. [PubMed: 17682663]

Buysse DJ, Reynolds CF, Monk TH, Berman SR, Kupfer DJ. The Pittsburgh Sleep Quality Index: A new instrument for psychiatric practice and research. Psychiatry Research. 1989; 28:193-213. [PubMed: 2748771]

Castelloe, JM., Zimmerman, DL. Technical Report. Department of Statistics and Actuarial Science, University of Iowa; 2002. Convergence assessment for reversible jump MCMC samplers; p. 313

Dahlhaus R. Fitting time series models to nonstationary processes. The Annals of Statistics. 1997; 25:1-37.

Davis R, Lee T, Rodriguez-Yam G. Structural break estimation for nonstationary time series models. Journal of American Statistical Association. 2006; 101:223-239.

Diggle PJ, Al Wasel I. Spectral analysis of replicated biomedical time series. Applied Statistics. 1997:31-71.

Fiecas M, Ombao H. Modeling the evolution of dynamic brain processes during an associative learning experiment. Journal of American Statistical Association. 2016; 111:1440-1453.

Gu, C. Smoothing spline ANOVA models. Springer; New York: 2013.

Guo W, Dai M, Ombao HC, von Sachs R. Smoothing spline ANOVA for time-dependent spectral analysis. Journal of the American Statistical Association. 2003; 98:643-652.

Hall M, Vasko R, Buysse D, Ombao H, Chen Q, Cashmere JD, Kupfer D, Thayer JF. Acute stress affects heart rate variability during sleep. Psychosomatic Medicine. 2004; 66:56-62. [PubMed: 14747638]

Iber, C., Ancoli-Isreal, S., Chesson, A., Quan, SF. The AASM Manual for the Scoring of Sleep and Associated Events. American Academy of Sleep Medicine; Westchester, I. L: 2007.

Jurysta F, van de Borne P, Migeotte P, Dumont M, Lanquart J, Degaute J, Linkowski P. A study of the dynamic interactions between sleep EEG and heart rate variability in healthy young men. Clinical Neurophysiology. 2003; 114:2146-2155. [PubMed: 14580613]

Krafty RT. Discriminant analysis of time series in the presence of within-group spectral variability. Journal of Time Series Analysis. 2016; 37:435-450. [PubMed: 27695143]

Krafty RT, Hall M, Guo W. Functional mixed effects spectral analysis. Biometrika. 2011; 98:583-598. [PubMed: 26855437] 
Malik M, Camm AJ, Bigger JT Jr, Breithardt G, Cerutti S, Cohen RJ, Coumel P, Fallen EL, Kennedy HL, Kleiger RE, Lombardi F, Malliani A, Moss AJ, Rottman JN, Schmidt G, Schwartz PJ, Singer DH. Heart rate variability. standards of measurement, physiological interpretation, and clinical use. European Heart Journal. 1996; 17:354-381. [PubMed: 8737210]

McCurry SM, Logsdon RG, Teri L, Vitiello MV. Sleep disturbances in caregivers of persons with dementia: Contributing factors and treatment implications. Sleep Medicine Reviews. 2007; 11:143-153. [PubMed: 17287134]

Ombao H, von Sachs R, Guo W. SLEX analysis of multivariate nonstationary time series. Journal of the American Statistical Association. 2005; 100:519-531.

Pawitan Y, O'Sullivan F. Nonparametric spectral density estimation using penalized Whittle likelihood. Journal of the American Statistical Association. 1994; 89:600-610.

Priestley MB. Evolutionary spectra and non-stationary process. Journal of the Royal Statistical Society, Series B. 1965; 28:228-240.

Qin L, Guo W, Litt B. A time-frequency functional model for locally stationary time series data. Journal of Computational and Graphical Statistics. 2009; 18:675-693. [PubMed: 20228961]

Qin L, Wang Y. Nonparametric spectral analysis with applications to seizure characterization using eeg time series. The Annals of Applied Statistics. 2009; 2:1432-1451.

Rosen O, Wood S, Stoffer DS. AdaptSPEC: Adaptive spectral estimation for nonstationary time series. Journal of the American Statistical Association. 2012; 107:1575-1589.

Schwarz K, Krivobokova T. A unified framework for spline estimators. Biometrika. 2016; 103:121131.

Vanoli E, Adamson PB, Ba-Lin, Pinna GD, Lazzara R, Orr WC. Heart rate variability during specific sleep stages. Circulation. 1995; 91:1918-1922. [PubMed: 7895347]

Whittle P. Curve and periodogram smoothing. Journal of the Royal Statistical Society. Series B (Methodological). 1957; 19:38-63.

Yang WH, Holan SH, Wikle CK. Bayesian lattice filters for time-varying autoregression and timefrequency analysis. Bayesian Analysis. 2016; 11:977-1003. 

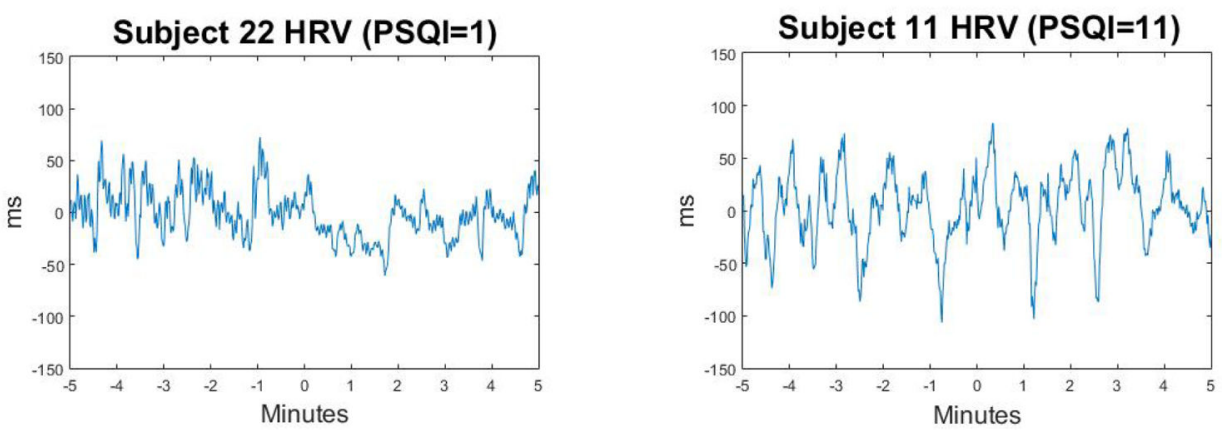

Figure 1.

Demeaned heart rate variability time series 5 minutes before and 5 minutes after the first onset of REM sleep for two study participants. PSQI scores for the participants are 1 and 11, respectively, indicating worsening sleep quality. 


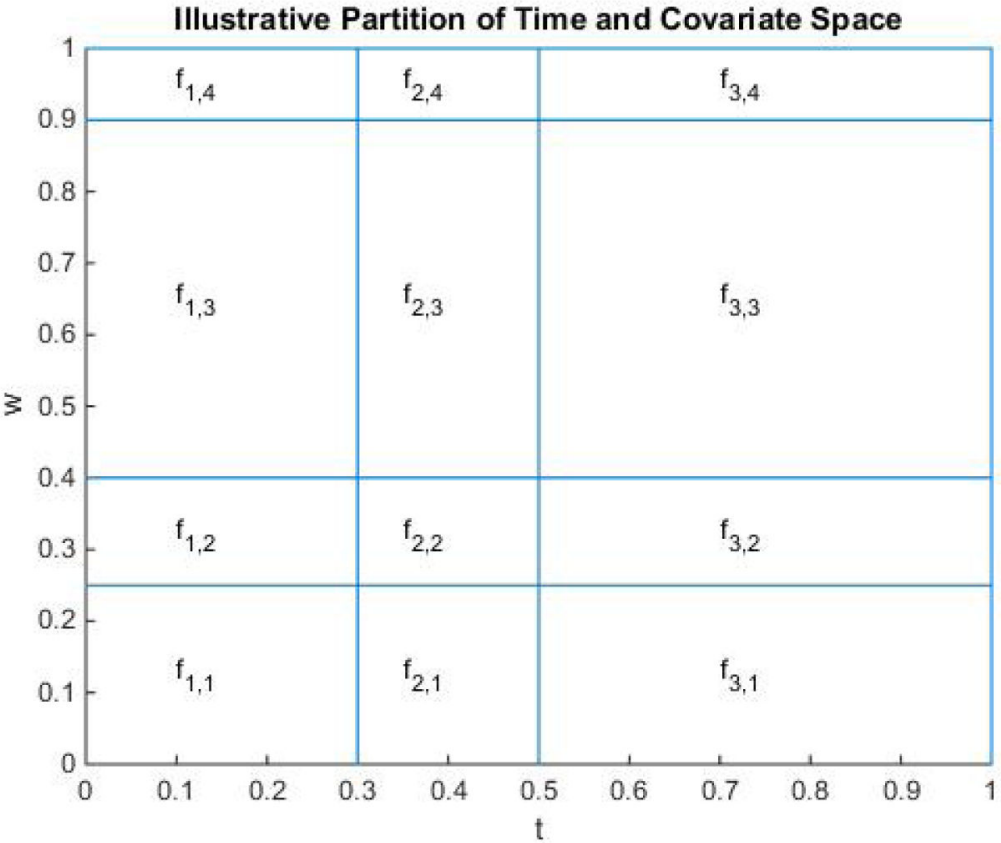

Figure 2.

Illustration of a partition of the time (t) and covariate (w) space into 3 time segments, $\left(\xi_{1}\right.$, $\left.\xi_{2}, \xi_{3}\right)^{\prime}$, and 4 covariate segments $\left(\psi_{1}, \psi_{2}, \psi_{3}, \psi_{4}\right)^{\prime}$, and the power spectra for all 12 blocks making up the partition. 


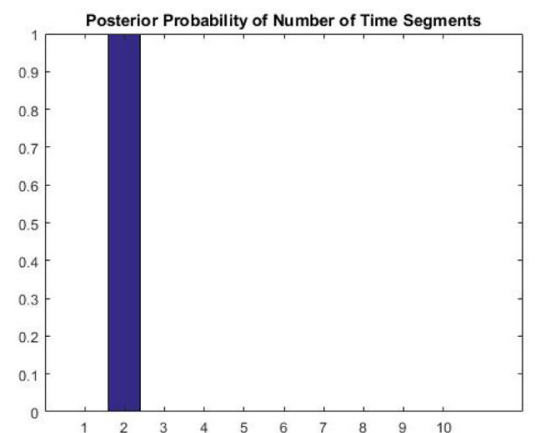

(a)

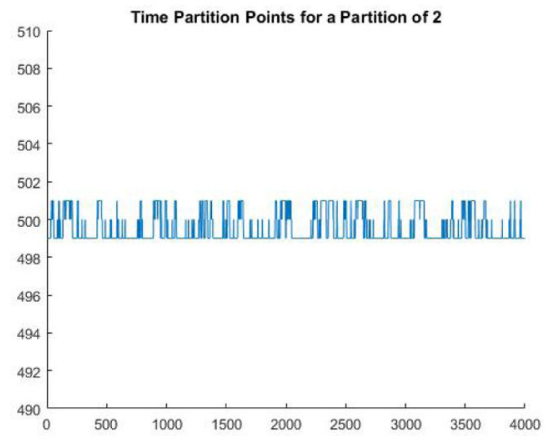

(c)

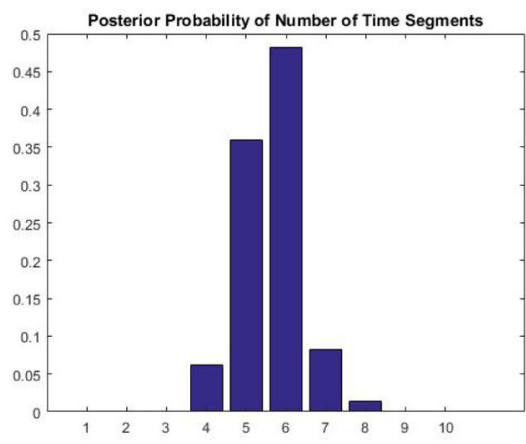

(b)

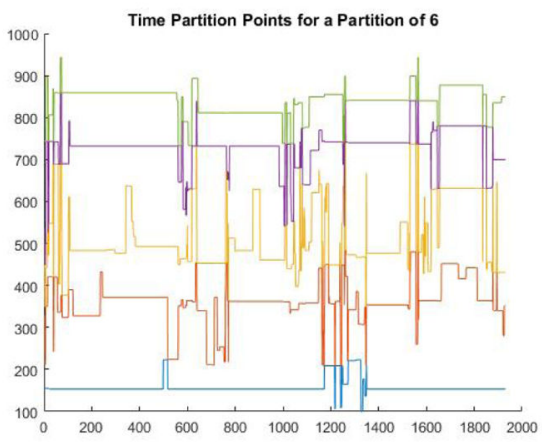

(d)

Figure 3.

The first row contains posterior probabilities for the number of time segments for the piecewise AR process (a) and the slowly varying AR process (b). The second row contains the time-varying distribution of time partition points for iterations with two time segments for the piecewise AR process (c) and six time segments for the slowly varying AR process (d). The $y$-axis of plot (c) has been restricted to show the movement of the sampler around the true partition point. 


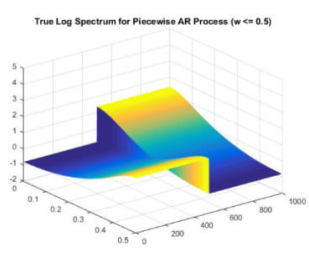

(a)

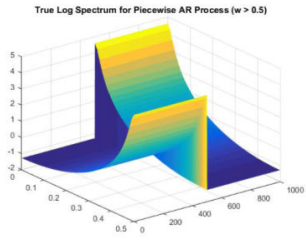

(c)

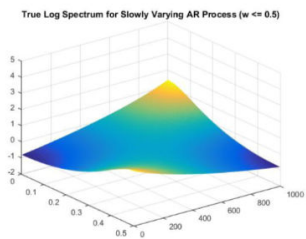

(e)

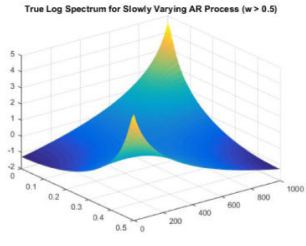

(g)

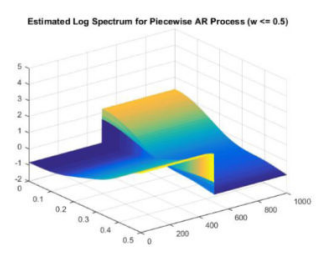

(b)

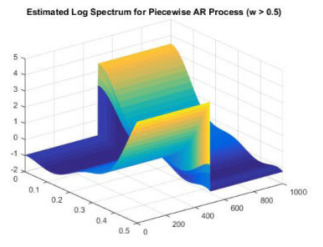

(d)

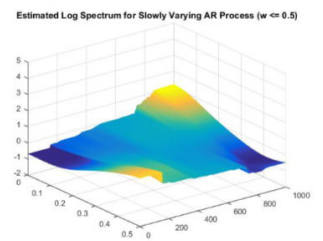

(f)

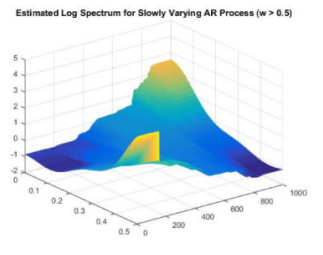

(h)

Figure 4.

The first two rows contain the true $(\mathrm{a}, \mathrm{c})$ and estimated average $(\mathrm{b}, \mathrm{d})$ time-varying log spectra for the covariate modulated piecewise AR process, where $w \ell \leq 0.5$ and $w p>0.5$. The last two rows contain the true (e, g) and estimated average (f, h) time-varying log spectra for the covariate-modulated slowly varying AR process, where $w \ell \leq 0.5$ and $w \ell>0.5$. 
Time-Varying Log Spectrum for PSOI 1-3

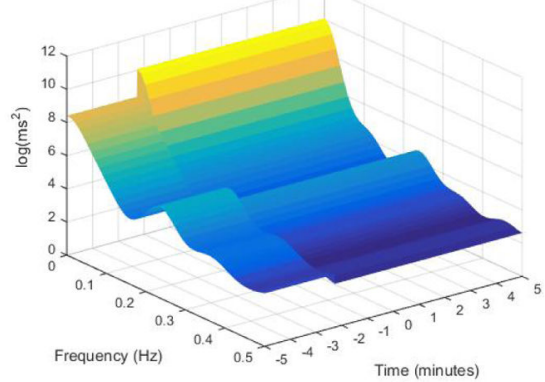

Time-Varying Log Spectrum for PSQI 11-13

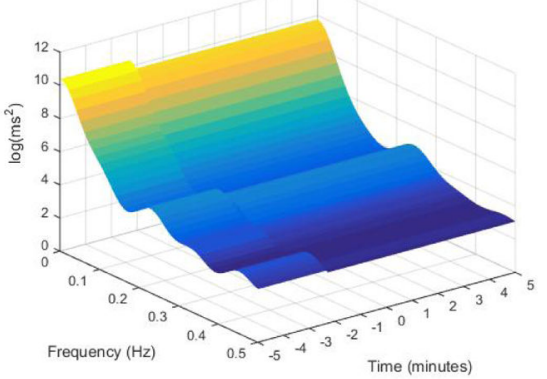

Figure 5.

Estimated average time-varying log spectra for the 5 minutes before and 5 minutes after the first period of rapid eye movement sleep for PSQI scores 1-3 and 11-13 respectively. 


\section{Table 1}

The mean (standard deviation) of the mean square error of the estimators of the log spectrum from 100 random samples of collections of piecewise and slowly varying AR processes. Two estimation procedures are implemented: CABS, the proposed conditional adaptive Bayesian spectrum estimator; GCV, ad hoc procedure partitioning in time and covariate and smoothing in frequency using generalized cross validation. The number of equally spaced samples in scaled time $(T)$ and scaled covariate $(L)$ are varied to show how the estimation accuracy changes as sampling rates increase.

\begin{tabular}{|c|c|c|c|c|}
\hline Change & $\boldsymbol{T}$ & $\boldsymbol{L}$ & CABS & GCV \\
\hline \multirow{5}{*}{ Piecewise } & 1000 & 20 & $0.1190(0.0580)$ & $1.0901(0.0371)$ \\
& 1000 & 40 & $0.1130(0.0598)$ & $1.0841(0.0260)$ \\
& 1000 & 80 & $0.1079(0.0557)$ & $1.0856(0.0189)$ \\
\hline \multirow{5}{*}{2000} & 20 & $0.0748(0.0252)$ & $0.8624(0.0253)$ \\
& 2000 & 40 & $0.0755(0.0274)$ & $0.8627(0.0205)$ \\
& 2000 & 80 & $0.0702(0.0230)$ & $0.8620(0.0119)$ \\
\hline \multirow{5}{*}{ Slowly Varying } & 4000 & 20 & $0.0617(0.0121)$ & $0.5677(0.0138)$ \\
& 4000 & 40 & $0.0610(0.0133)$ & $0.5660(0.0109)$ \\
& 4000 & 80 & $0.0607(0.0135)$ & $0.5679(0.0077)$ \\
& 1000 & 20 & $0.0353(0.0052)$ & $1.0588(0.0381)$ \\
& 1000 & 40 & $0.0260(0.0031)$ & $1.0569(0.0241)$ \\
& 1000 & 80 & $0.0206(0.0026)$ & $1.0580(0.0186)$ \\
& 2000 & 80 & $0.0148(0.0013)$ & $0.7596(0.0116)$ \\
\hline & 4000 & 20 & $0.0177(0.0019)$ & $0.5548(0.0152)$ \\
& 4000 & 40 & $0.0137(0.0012)$ & $0.5546(0.0115)$ \\
& 4000 & 80 & $0.0110(0.0009)$ & $0.5546(0.0068)$ \\
\hline
\end{tabular}

